\title{
A PUZZLE INVOLVING GALACTIC BULGE MICROLENSING EVENTS
}

This article has been downloaded from IOPscience. Please scroll down to see the full text article.

2010 ApJ 711 L48

(http://iopscience.iop.org/2041-8205/711/1/L48)

The Table of Contents and more related content is available

Download details:

IP Address: 131.215.193.213

The article was downloaded on 26/03/2010 at 21:27

Please note that terms and conditions apply. 


\title{
A PUZZLE INVOLVING GALACTIC BULGE MICROLENSING EVENTS*
}

\author{
Judith G. Cohen ${ }^{1}$, Andrew Gould ${ }^{2}$, Ian B. Thompson ${ }^{3}$, Sofia Feltzing ${ }^{4}$, Thomas Bensby ${ }^{5}$, Jennifer A. Johnson ${ }^{2}$, \\ Wenjin Huang ${ }^{6,11}$, Jorge Meléndez 7 , Sara Lucatello ${ }^{8,9}$, and Martin Asplund ${ }^{10}$ \\ ${ }^{1}$ Palomar Observatory, Mail Stop 249-17, California Institute of Technology, Pasadena, CA 91125, USA; jlc@ astro.caltech.edu \\ ${ }^{2}$ Department of Astronomy, Ohio State University, 140 W. 18th Ave., Columbus, OH 43210, USA; gould@ astronomy.ohio-state.edu, jaj@astronomy.ohio-state.edu \\ ${ }^{3}$ Carnegie Observatories, 813 Santa Barbara Street, Pasadena, CA 91101, USA; ian@obs.carnegiescience.edu \\ ${ }^{4}$ Lund Observatory, Box 43, SE-22100 Lund, Sweden; sofia@astro.lu.se \\ ${ }^{5}$ European Southern Observatory, Alonso de Cordova 3107, Vitacura, Casilla 19001, Santiago 19, Chile; tbensby@eso.org \\ ${ }^{6}$ Palomar Observatory, Mail Stop 105-24, California Institute of Technology, Pasadena, CA 91125, USA; hwenjin@ astro.washington.edu \\ ${ }^{7}$ Centro de Astrofísica da Universidade do Porto, Rua das Estrelas, 4150-762 Porto, Portugal; jorge @ astro.up.pt \\ ${ }^{8}$ INAF-Astronomical Observatory of Padova, Vicolo dell'Osservatorio 5, 35122 Padova, Italy; sara.lucatello@oapd.inaf.it \\ ${ }^{9}$ Excellence Cluster Universe, Technische Universität München, Boltzmannstr. 2 D-85748 Garching, Germany \\ ${ }^{10}$ Max Planck Institute for Astrophysics, Postfach 1317, 85741 Garching, Germany; asplund@MPA-Garching.MPG.DE \\ Received 2009 November 26; accepted 2010 January 27; published 2010 February 11
}

\begin{abstract}
We study a sample of 16 microlensed Galactic bulge main-sequence turnoff region stars for which high-dispersion spectra have been obtained with detailed abundance analyses. We demonstrate that there is a very strong and highly statistically significant correlation between the maximum magnification of the microlensed bulge star and the value of the $[\mathrm{Fe} / \mathrm{H}]$ deduced from the high resolution spectrum of each object. Physics demands that this correlation, assuming it to be real, be the result of some sample bias. We suggest several possible explanations, but are forced to reject them all, and are left puzzled. To obtain a reliable metallicity distribution in the Galactic bulge based on microlensed dwarf stars, it will be necessary to resolve this issue through the course of additional observations.
\end{abstract}

Key words: Galaxy: bulge - gravitational lensing: micro - stars: abundances

Online-only material: machine-readable table

\section{INTRODUCTION}

Microlensing occurs when a "lens" (star, planet, black hole, etc.) becomes closely aligned with a more distant "source" star, whose image is both magnified and distorted by the gravitational field of the "lens." Microlensing of stars in the Galactic bulge offers the possibility of studying in detail stars that are too faint for such even with the largest existing telescopes without a microlensing boost. The lens is usually a star in the Galactic bulge, but sometimes in the disk of the Milky Way (Dominik 2006).

Bulge giants are bright enough that high-dispersion spectra can routinely be obtained with $8 \mathrm{~m}$ class telescopes. Extensive surveys of such giants at optical wavelengths (see, e.g., Fulbright et al. 2006) have been carried out to construct the metallicity distribution function (MDF) for the bulge, many of them in Baade's window $\left(b \sim-4^{\circ}\right)$, the innermost field of relatively low reddening. Zoccali et al. (2008) have presented results of a survey of $[\mathrm{Fe} / \mathrm{H}]$ in the Galactic bulge from spectra of about 800 stars. They find a radial gradient in $[\mathrm{Fe} / \mathrm{H}]$ within the bulge with the mean value going from +0.03 dex at $b=-4^{\circ}$ to -0.12 dex at $b=-6^{\circ}$ and a sharp cutoff toward higher metallicities with less than $5 \%$ of the sample in Baade's window having $[\mathrm{Fe} / \mathrm{H}]>0.4 \mathrm{dex}$. Rich et al. (2007), who reach into $(l, b)=\left(0^{\circ},-1^{\circ}\right)$ with high dispersion in the near-IR, still find a sub-solar mean metallicity of $-0.22 \pm 0.01$ dex.

The ability to obtain high resolution, high-quality spectra of microlensed Galactic bulge dwarfs and to carry out a detailed

\footnotetext{
* Based in part on observations obtained at the W. M. Keck Observatory, which is operated jointly by the California Institute of Technology, the University of California, and the National Aeronautics and Space Administration.

${ }^{11}$ Current address: Department of Astronomy, University of Washington, Box 351580, Seattle, WA 98195-1580, USA
}

abundance analysis offers an independent apparently unbiased way to determine the MDF of Galactic bulge stars, as well as their detailed chemical inventory. In principle, the abundance analysis of an upper main-sequence dwarf is much easier and less prone to error for spectra of a fixed signal-to-noise ratio $(\mathrm{S} / \mathrm{N})$ than that of a much cooler but much brighter bulge giant with a very complex spectrum full of blends and strong molecular bands.

\section{THE SAMPLE OF MICROLENSED GALACTIC BULGE STARS}

We consider here the sample of subgiants near the mainsequence turnoff (MSTO), stars at the MSTO, or dwarfs of slightly lower luminosity than the MSTO which have had high-magnification microlensing events during which highdispersion spectra have been obtained with large optical telescopes. We denote this group as the microlensed MSTO dwarfs in the Galactic bulge. Our sample includes all such stars with published abundance analyses.

The pioneering work of Cavallo et al. (2003) ${ }^{12}$ includes three such stars, MACHO-98-BLG-6, MACHO-99-BLG-1,13 and MACHO-99-BLG22; see, e.g., Alcock et al. (1999) for a description of the MACHO survey. Johnson et al. (2007) (MOA2006-BLG265) were the first to piggy-back on the microlensing planet hunters, who prize high-magnification events because of their extreme sensitivity to planets (Udalski et al. 2005; Gould et al. 2006; Gaudi et al. 2008). This star proved to be extremely metal-rich, much more so than the bulk of the much larger

\footnotetext{
12 Lennon et al. $(1996,1997)$ presented earlier analyses of low-dispersion spectra of three such bulge stars which are broadly consistent with the correlation shown in Figure 1.

13 We omit this star as its spectrum shows double lines according to Bensby et al. (2010).
} 
Table 1

Data for Microlensed MSTO Bulge Stars

\begin{tabular}{lcrrr}
\hline \hline \multicolumn{1}{c}{ Name } & Obs. Code & $A_{\max }$ & $A_{\mathrm{obs}} \mathrm{b}^{\mathrm{b}}$ & $\begin{array}{r}{[\mathrm{Fe} / \mathrm{H}]^{\mathrm{c}}} \\
(\mathrm{dex})\end{array}$ \\
\hline MACHO-1998-BLG-6 & $\mathrm{K}$ & 5 & 4 & -0.22 \\
MACHO-1999-BLG-22 & $\mathrm{K}$ & 28 & 12 & -0.35 \\
MOA-2006-BLG99 & $\mathrm{M}$ & 515 & 110 & +0.36 \\
OGLE-2006-BLG265S & $\mathrm{K}$ & 210 & 135 & +0.56 \\
OGLE-2007-BLG349S & $\mathrm{K}$ & 450 & 400 & +0.56 \\
OGLE-2007-BLG514S & $\mathrm{M}$ & 1000 & 500 & +0.33 \\
OGLE-2008-BLG209 & $\mathrm{M}$ & 30 & 22 & -0.33 \\
MOA-2008-BLG-311 & $\mathrm{M}$ & 285 & 200 & +0.26 \\
MOA-2008-BLG-310S & $\mathrm{M}$ & 380 & 313 & +0.42 \\
MOA-2009-BLG259 & $\mathrm{K}$ & 223 & 223 & +0.55 \\
OGLE-2009-BLG-076S & $\mathrm{V}$ & 68 & 48 & -0.76 \\
MOA-2009-BLG-493 & $\mathrm{V}$ & 150 & 123 & -0.71 \\
MOA-2009-BLG-133 & $\mathrm{V}$ & 74 & 35 & -0.67 \\
MOA-2009-BLG-475 & $\mathrm{V}$ & 62 & 48 & -0.54 \\
MOA-2009-BLG-489 & $\mathrm{V}$ & 103 & 103 & -0.18 \\
MOA-2008-BLG-456 & $\mathrm{V}$ & 77 & 47 & +0.12 \\
\hline
\end{tabular}

Notes.

${ }^{a}$ M: Magellan; K: Keck; V: VLT.

b Magnification at the time the spectroscopic observations were carried out.

${ }^{\mathrm{c}}$ References for each star are given in Section 2.

samples of bulge giants; the unusually high metallicity aroused considerable interest in the astronomical community. Improvements in the current generation of microlensing surveys of the bulge, the OGLE collaboration ${ }^{14}$ (Udalski 2003) and the Microlensing Observations in Astrophysics (MOA) collaboration ${ }^{15}$ (Bond et al. 2002), led to increasing numbers of alerts; they together find a total of about 800 microlensing events per year, of which the Microlensing Follow Up Network $(\mu \mathrm{FUN})^{16}$ is able to identify about 10 as high-magnification events.

Recognition of the importance of observations of transient sources has led to modifications in telescope operations to enhance our ability to take advantage of such brief opportunities. Those advances enabled the work of Johnson et al. (2008) (MOA-2006-BLG99), Cohen et al. (2008) (OGLE-2007-BLG349S), the latter spectrum with signal-to-noise ratio/spectral resolution element $(\mathrm{S} / \mathrm{N})>90$ for $\lambda>5500 \AA$, and of Cohen et al. (2009) (MOA-2008-BLG-310S and 311S), as well as the very recently completed analysis of OGLE-2007-BLG514S (Epstein et al. 2010). Each of these microlensed MSTO bulge stars turned out to have very high $[\mathrm{Fe} / \mathrm{H}]$. The first three of these led to the suggestion by Cohen et al. (2008) that the true MDF in the Galactic bulge was that of the dwarfs, characterized by significantly higher mean $[\mathrm{Fe} / \mathrm{H}]$ than that of the giant bulge samples.

All of these stars were observed with either HIRES (Vogt et al. 1994) at the Keck I telescope or the MIKE spectrograph (Bernstein et al. 2003) at the $6.5 \mathrm{~m}$ Magellan Clay Telescope at the Las Campanas Observatory. Last year, a group led by S. Feltzing, T. Bensby, and J. Johnson began observing MSTO microlensed bulge stars with UVES (Dekker et al. 2000) at the Very Large Telescope (VLT). Results from two stars, OGLE2008-BLG-209S (Bensby et al. 2009a, a MIKE spectrum) and OGLE-2009-BLG-076S (Bensby et al. 2009b), followed, both of which were metal-poor, with $[\mathrm{Fe} / \mathrm{H}]-0.33$ and $-0.76 \mathrm{dex}$, respectively. Bensby et al. (2010) suggested that the previous

\footnotetext{
14 http://www.astrouw.edu.pl/ ogle/ogle3/ews/ews.html

15 http://www.phys.canterbury.ac.nz/moa

$16 \mathrm{http}: / /$ www.astronomy.ohio-state.edu/ microfun/
}

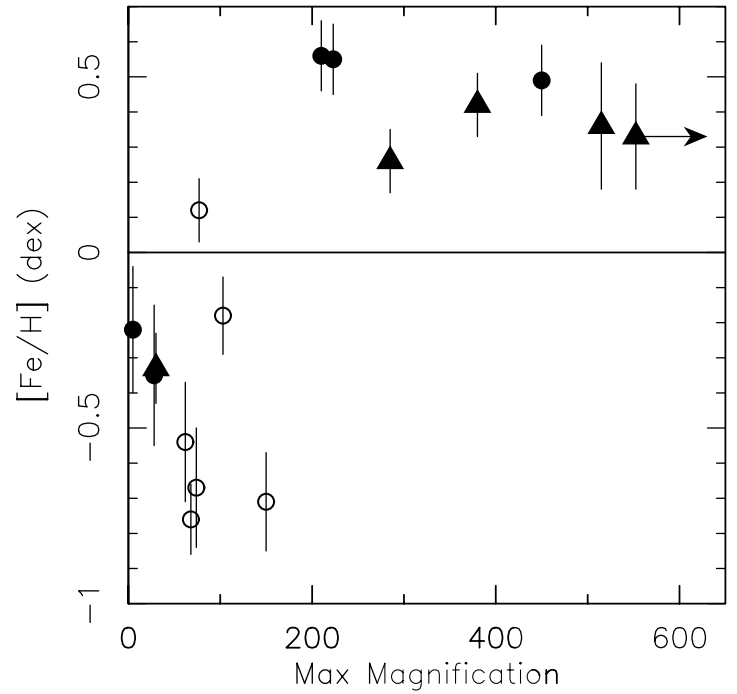

Figure 1. $[\mathrm{Fe} / \mathrm{H}]$ for the sample of 16 microlensed MSTO Galactic bulge stars with detailed abundance analyses is shown as a function of the maximum magnification achieved in each lensing event. Filled circles denote the Keck sample, filled triangles the Magellan sample, and open circles denote those with VLT spectra.

high metallicities for microlensed bulge dwarfs might be just a matter of chance; they find that they cannot reject the possibility that the MDFs for the bulge giants and microlensed dwarfs are identical.

Here, we adopt the $[\mathrm{Fe} / \mathrm{H}]$ and their uncertainties given in the published papers referenced above. To this sample we add MOA-2009-BLG259S, observed with HIRES/Keck in 2009 July for which J. G. Cohen et al. (2010, in preparation) find $[\mathrm{Fe} / \mathrm{H}]=+0.55 \pm 0.10 \mathrm{dex}$. We also add five microlensed bulge dwarfs observed with the VLT from Bensby et al. (2010) for a total sample of 16 microlensed MSTO Galactic bulge stars.

\section{THE MAXIMUM MAGNIFICATION OF THE MICROLENSING EVENTS}

The maximum magnification, $A_{\max }$, of each of these microlensing events is based for all recent events on fits to high cadence photometry obtained by $\mu$ FUN. ${ }^{17}$ Poindexter et al. (2005) give $A_{\max }$ for the MACHO events; we use Janczak et al. (2009) for MOA-2008-BLG-310S. $A_{\max }$, the ratio of the apparent brightness at the peak of the microlensing event to that before or after, is independent of reddening. It ranges from 5 to $\sim 1000$ for our sample stars (see Table 1). Figure 1 shows the relationship between $A_{\max }$ for the event and $[\mathrm{Fe} / \mathrm{H}]$ of the source derived from the high-dispersion spectra for each of the 16 microlensed Galactic bulge stars in our sample.

It is apparent from Figure 1 that there is a very strong correlation between $A_{\max }$ and $[\mathrm{Fe} / \mathrm{H}]$ for the MSTO sample of microlensed bulge stars. A similarly strong correlation is shown when $A$ at the time the spectra were acquired is used instead. A Spearman rank test indicates that the two-sided probability that $A_{\max }$ is not correlated with $[\mathrm{Fe} / \mathrm{H}]$ is $5 \times 10^{-3}$. If MACHO-1998BLG6, which Bensby et al. (2010) consider a low-luminosity giant, not a subgiant, is omitted, the two-sided probability for the remaining 15 stars becomes $6 \times 10^{-3}$, not significantly larger. While in principle this could be a statistical fluke, the formal probability of this is sufficiently low to investigate the implications of it being a real effect.

\footnotetext{
17 The $A_{\max }$ from the MOA Web site are preliminary values only.
} 


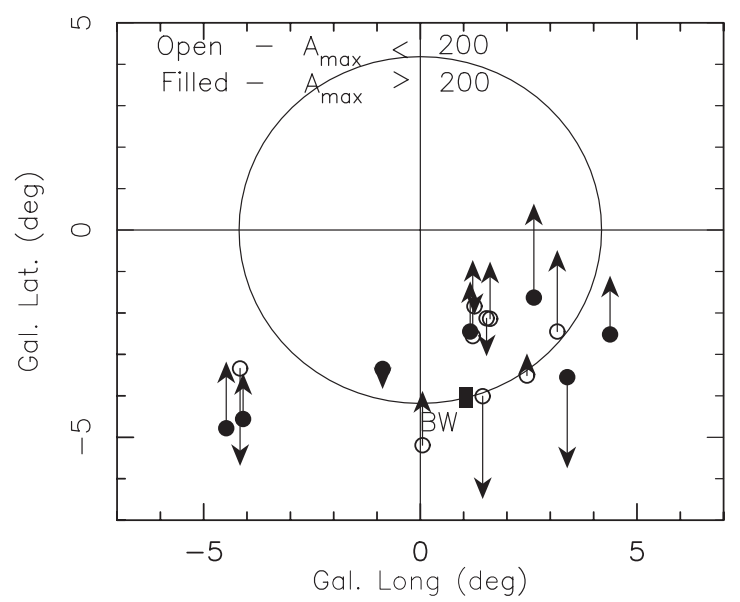

Figure 2. Distribution in Galactic latitude and longitude of the sample of 16 microlensed MSTO bulge stars. The heliocentric radial velocity for each star is indicated by an arrow, upward being positive, with a scale of $70 \mathrm{~km} \mathrm{~s}^{-1}$ per degree. Baade's window is marked by the filled rectangle, and its Galactocentric radius is indicated by a circle.

Figure 2 shows the locations on the sky of the sample of microlensed bulge stars. The region of positive Galactic latitude in general has significantly larger extinction than that of negative $b$ in the region of the Galactic center, and much of that area is not covered by any microlensing survey. There is no obvious difference in the projected spatial distribution on the sky of the very high versus the lower $A_{\max }$ bulge microlensed MSTO stars. The maximum projected distance from the Galactic center for a star in our sample is $6.6(0.9 \mathrm{kpc})$, the minimum 2.2 ; the median is 4.0 , the same as that of Baade's window.

Thus in addition to the discrepancy between the MDF of the bulge giants versus that of the microlensed dwarfs reviewed in Section 1, an even more puzzling and potentially more serious problem is introduced by Figure 1. At first glance, this figure suggests the presence of two populations, one at $[\mathrm{Fe} / \mathrm{H}]$ about +0.35 dex with $\sigma([\mathrm{Fe} / \mathrm{H}])$ small and with $A_{\max }>200$, and one with mean $[\mathrm{Fe} / \mathrm{H}]$ considerably lower at about -0.4 dex, a larger dispersion in metallicity, and with $A_{\max }<200$. Since we have already shown that the projected distributions of the low- and high-magnification events are similar, we next consider systematically different positions along the line of sight. One might imagine that spatially separating the high and low metallicity microlensing events, having them arise in regions of different stellar density (presumably closer or further from the Galactic center) in the presence of radial gradients in $[\mathrm{Fe} / \mathrm{H}]$ within the bulge, could lead to the very strong correlation seen in Figure 1.

Microlensing with stars both as sources and lenses is a phenomenon depending only on geometry (i.e., distances and impact parameter) and the mass of the lens. An ensemble of microlensing events also has properties that depend on the spatial density of the sources and lenses. The distribution in magnification for a particular source and a particular lens always has a much higher probability for events with large impact parameter (low $A_{\max }$ ) than it does for events with small impact parameter (high-magnification events). The absence of any event with high $[\mathrm{Fe} / \mathrm{H}]$ and $A_{\max }<200$, given the many very high $A_{\max }$, high $[\mathrm{Fe} / \mathrm{H}]$ events we see, poses an insurmountable problem to any hypothesis that seeks to explain the trend seen in Figure 1 through a mechanism of spatially separate populations within the bulge with differing mean metallicities, densities, etc.
If the basic physical laws governing microlensing are not to be violated, there must be a previously unrecognized sample bias that produces the strong correlation between $A_{\max }$ and $[\mathrm{Fe} / \mathrm{H}]$ seen in Figure 1. We consider several possibilities below.

1. When the angular size of the impact parameter for the microlensing event is so small that it becomes comparable to the angular size of the source star's radius, finite source effects, i.e., differential magnification of the limb relative to that of the center of the disk of the source star, become important. This occurs only in very high $A_{\max }$ events and affects the strength of spectral lines, which could potentially produce a spurious $[\mathrm{Fe} / \mathrm{H}]$ in the highest $A_{\max }$ events, leading to a systematic sample bias. Johnson et al. (2010) carried out detailed abundance studies of synthetic spectra of highly magnified dwarf stars. They concluded that the effects are always less than 0.05 dex, which is much too small to produce the effect seen in Figure 1.

2. Perhaps in the very crowded fields of the Galactic bulge, the spectra in low-magnification events include a substantial contribution from close neighbors of the microlensed dwarf, while when $A_{\max }$ is large, spectroscopic observations only detect the source star. We have evaluated the contribution of blending stars by comparing the unlensed (baseline) brightness inferred from the microlensing light curve with that from OGLE or MOA images long before the event. ${ }^{18}$ In the worst case (MOA-2009-BLG493), there is a $10 \%$ contribution at the time of observation, the second worst case has a 5\% contribution by blending stars; for all other events this is not an issue. This demonstrates that blending is not the answer. Furthermore double-lined spectra are not seen among any of these microlensed dwarfs.

3. Perhaps for some of the events the source is a foreground disk star. If disk stars have a lower mean $[\mathrm{Fe} / \mathrm{H}]$ than that of the bulge and if such events preferentially include those with low $A_{\max }$, this could reproduce the observed relationship shown in Figure 1. However, extrapolating the linear fit to the metallicity gradient determined by Luck et al. (2006) outside $4 \mathrm{kpc}$ from the center further inward, the disk would reach $[\mathrm{Fe} / \mathrm{H}]+0.5$ dex at $R_{\mathrm{GC}}=1 \mathrm{kpc}$. In addition, calculations of the probability of microlensing for a source in the Galactic disk (see, e.g., Kane \& Sahu 2006) demonstrate that the source of a microlensing event toward the bulge actually be a foreground disk star is unlikely. Furthermore, the measured radial velocities show that the sources of these microlensing events are a kinematically hot population with $\sigma\left(v_{r}\right)$ much too large for the Galactic disk; see Figure 2. Additional arguments supporting a bulge origin for the MSTO microlensing dwarfs are given by, e.g., Bensby et al. (2010).

4. Perhaps the fault lies in systematic errors in $[\mathrm{Fe} / \mathrm{H}]$. There is a real sampling bias among the active groups working in this area. The initial results of the analyses of two microlensed MSTO bulge stars by Johnson et al. (2007, 2008) yielded surprisingly high metallicities. Even solartype dwarfs with such high $[\mathrm{Fe} / \mathrm{H}]$ have complex spectra, with many blended and overlapping features, and the photometry is not trustworthy in regions with such high and variable reddening. Cohen and Thompson, who lead the efforts at the Keck and Magellan Observatories, believed that such a controversial issue could only be finally settled on the basis of very high signal-to-noise spectra. This meant

\footnotetext{
18 The required data are not easily available for the MACHO events.
} 
Table 2

Check of 2008 OGLE Bulge Microlensing Alerts

\begin{tabular}{lcccc}
\hline \hline $\begin{array}{c}\text { OGLE } \\
\text { ID Number }\end{array}$ & $u_{0}$ & $\begin{array}{c}t_{E} \\
\text { (days) }\end{array}$ & $\begin{array}{c}I \text { (source) } \\
(\mathrm{mag})\end{array}$ & Code $^{\mathrm{a}}$ \\
\hline 2 & 0.4110 & 16.767 & 17.187 & 1 \\
3 & 0.0123 & 99.967 & 21.882 & 2 \\
4 & 0.1500 & 17.761 & 16.574 & 2 \\
5 & 0.5054 & 17.630 & 14.734 & 2 \\
6 & 0.2840 & 71.667 & 19.505 & 2 \\
9 & 0.7880 & 28.962 & 18.509 & 1 \\
10 & 0.0830 & 72.709 & 19.536 & 1 \\
11 & 0.2990 & 44.934 & 17.364 & 1 \\
13 & 0.0230 & 74.357 & 16.206 & 1 \\
14 & 0.3030 & 23.270 & 18.468 & 1 \\
15 & 1.1050 & 6.526 & 17.918 & 1 \\
\hline
\end{tabular}

Notes.

a 1 : original values unchanged; 2 : altered.

(This table is available in its entirety in a machine-readable form in the online journal. A portion is shown here for guidance regarding its form and content.)

that they triggered target-of-opportunity observations only for the brightest of the microlensed bulge dwarfs, which tend to be those with the highest $A_{\max }$. The VLT group has observed events with a larger range of brightness, and hence of $A_{\max }$.

In light of the sample bias between the VLT and the Magellan/Keck groups, the relevant question is whether the $[\mathrm{Fe} / \mathrm{H}]$ values determined by the various groups involved are on a consistent scale. Since the low-magnification events are in general fainter, one might expect the resulting spectra to be on average of lower quality with lower S/Ns. Given that line crowding and blending make the definition of the continuum in these spectra difficult, this might produce a bias of underestimating $[\mathrm{Fe} / \mathrm{H}]$ in the required sense.

Bensby et al. (2009a; 2010) present comparisons of multiple independent analyses by J. Johnson, J. G. Cohen, and T. Bensby, and collaborators of HIRES or Mike spectra of six microlensed MSTO stars included in our sample. The deduced $[\mathrm{Fe} / \mathrm{H}]$ values are identical to within \pm 0.10 dex in all cases. Very recently, the two lowest $\mathrm{S} / \mathrm{N}$ spectra from the VLT sample, OGLE-2009-BLG-076S and MOA-2009BLG-475, were also analyzed by J. Cohen. Even for these very low $\mathrm{S} / \mathrm{N}$ spectra the derived $[\mathrm{Fe} / \mathrm{H}]$ values were in agreement to within the uncertainties, for the former being $-0.45 \pm 0.20$ versus $-0.72 \pm 0.12 \mathrm{dex}$ and for the latter $-0.49 \pm 0.20$ versus $-0.54 \pm 0.17$ dex .

We thus have established that there is good agreement in the derived $[\mathrm{Fe} / \mathrm{H}]$ from detailed abundance analyses when the three groups independently analyze the same spectrum of a microlensed MSTO bulge star. The remaining issue is whether $[\mathrm{Fe} / \mathrm{H}]$ derived from a spectrum of a microlensed MSTO bulge dwarf is independent of the $\mathrm{S} / \mathrm{N}$ within the range encompassed by our sample of 16 microlensed bulge MSTO stars. T. Bensby has carried out a test on the high S/N Keck spectrum of OGLE-2007-BLG349, degrading it to $\mathrm{S} / \mathrm{N} \sim 30$, and finds that the deduced $[\mathrm{Fe} / \mathrm{H}]$ changes by less than 0.05 dex. We emphasize that errors in $[\mathrm{Fe} / \mathrm{H}]$ arising from different analyses or from the $\mathrm{S} / \mathrm{N}$ of the observations appear to be too small to be the origin of the strong correlation between $A_{\max }$ and $[\mathrm{Fe} / \mathrm{H}]$ seen in Figure 1.

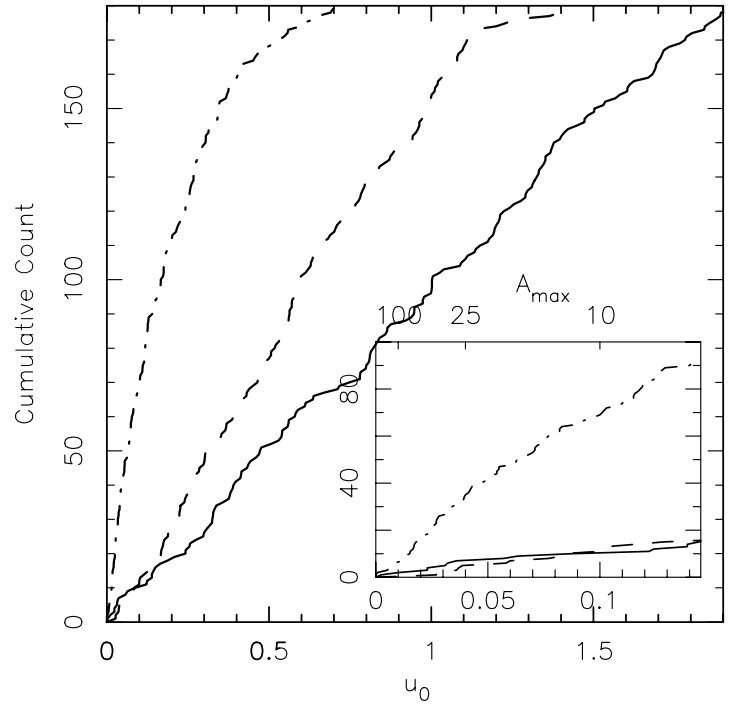

Figure 3. Cumulative counts of microlensing events as a function of $u_{0}$ (equivalent to $1 / A_{\max }$ for $A_{\max }>4$ ) for all OGLE alerts from 2008 that survived a check by hand for validity. Three different ranges of unlensed source brightness are shown: $I_{S}<17.93$ (solid line), $17.93<I_{s}<19.28$ (dashed line), and $I_{s}>19.28 \mathrm{mag}$ (dot-dashed line). Bin boundaries were chosen so that $1 / 3$ of the sample would be in each bin. For microlensing event selection by the OGLE survey to be independent of $A_{\max }$, this relation should be a straight line, as is shown for high magnification events in the inset.

5. There are some biases in the detectability of a microlensing event that depend on metallicity. These arise because the unlensed luminosity of a dwarf of a given mass and age is a function of metallicity. Tests with the $\mathrm{Y}^{2}$ isochrones (Kim et al. 2002) show that for a fixed age (we adopt $10 \mathrm{Gyr}$ ), the mass of a star at the turnoff is higher as metallicity increases and the turnoff becomes somewhat fainter in $M_{I}$. We adopt a Salpeter initial mass function and compare two populations with this age and with $[\mathrm{Fe} / \mathrm{H}]$ between -0.9 dex and +0.6 dex. While for a population with a fixed total mass, the total number of stars is significantly different in the two cases, the ratio of the number of stars on the upper red giant branch selected in a fixed range of $M_{I}$ to the number of stars in the turnoff region is approximately constant. Hence, this cannot explain differences in the MDF between the bulge giants and the bulge microlensed MSTO dwarfs nor lead to the correlation between $A_{\max }$ and $[\mathrm{Fe} / \mathrm{H}]$ seen in Figure 1.

6. Perhaps the procedures by which microlensing events are identified by the large surveys are biased in some way. This has been checked by A. Gould, who went through the entire set of OGLE microlensing alerts from 2008, examining each event, eliminating binaries, and redetermining $A_{\max }$ using all available photometry when necessary (see Table 2). He found that the number of microlensing alerts as a function of $A_{\max }$, equivalent to $1 / u_{0}$ for $A_{\max }>4$, has the form expected for selection of a sample unbiased in $A_{\max }$. Figure 3 shows the result, namely there is a linear relationship between cumulative counts and $u_{0}$ for $A_{\max }>15$. This is the expected relation for uniform completeness in the OGLE microlensing survey over each of the three ranges of unlensed source magnitude considered. The inset shows that the total number of events for $A_{\max }>15$ in each bin increases for fainter source stars, as expected, but this is because there are more faint stars 
than bright ones. Very high magnification events are very rare.

While we believe that the source of the very strong correlation seen in Figure 1 is some bias in the sample of microlensed bulge MSTO dwarfs, we have been unable to identify the source of the bias. Every mechanism that we have thought of can be ruled out with varying degrees of certainty. Unfortunately, until the sample bias for the microlensed MSTO bulge dwarfs is identified, the derived bulge-dwarf MDF and its comparison to the bulge-giant MDF must be treated very cautiously.

Although suitable high-magnification events are rare and lining up the necessary instruments/telescopes/clear weather at just the right time is difficult, with the Keck, Magellan, and VLT observatories all quite interested in this problem, the number of MSTO bulge microlensed dwarfs with high-dispersion spectra has risen rapidly and will continue to do so. But, assuming that the correlation between $A_{\max }$ of the event and $[\mathrm{Fe} / \mathrm{H}]$ of the source star continues to hold as the sample increases, what is really needed now just as urgently as larger samples is a new insight into what is causing the very strong correlation we have found between the maximum magnification of a microlensing event for bulge MSTO stars and their metallicities shown in Figure 1.

We are grateful to the many people who have worked to make the Keck Telescope and HIRES a reality and to operate and maintain the Keck Observatory. The authors wish to extend special thanks to those of Hawaiian ancestry on whose sacred mountain we are privileged to be guests. Without their generous hospitality, none of the observations presented herein would have been possible. We thank Chris Hirata and Sterl Phinney for helpful discussions. J.G.C. thanks NSF grants AST-0507219 and AST-0908139 for partial support. Work by A.G. was supported by NSF grant AST-0757888. I.B.T. thanks NSF grant AST0507325 for support.

\section{REFERENCES}

Alcock, C., et al. 1999, PASP, 111, 1539

Bensby, T., et al. 2009a, A\&A, 499, 737

Bensby, T., et al. 2009b, ApJ, 699, L174

Bensby, T., et al. 2010, A\&A, in press (arXiv:0911.5076)

Bernstein, R., Shectman, S. A., Gunnels, S. M., Mochnacki, S., \& Athey, A. E. 2003, Proc. SPIE, 4841, 1694

Bond, I., et al. 2002, MNRAS, 333, 71

Cavallo, R. M., Cook, K. H., Minniti, D., \& Vandehei, T. 2003, Proc. SPIE, 4834, 66

Cohen, J. G., Huang, W. J., Udalski, A., Gould, A., \& Johnson, J. A. 2008, ApJ, 682, 1029

Cohen, J. G., et al. 2009, ApJ, 699, 66

Dekker, H., et al. 2000, Proc. SPIE, 4008, 534

Dominik, M. 2006, MNRAS, 367, 669

Epstein, C. R., Johnson, J. A., Dong, S., Udalski, A., Gould, A., \& Becker, G. 2010, ApJ, 709, 447

Fulbright, J. P., McWilliam, A., \& Rich, R. M. 2006, ApJ, 636, 821

Gaudi, B. S., et al. 2008, Science, 315, 927

Gould, A., et al. 2006, ApJ, 644, L37

Janczak, J., et al. 2009, ApJ, 698, 1826

Johnson, J. A., Dong, S., \& Gould, A. 2010, ApJ, submitted (arXiv: astro-ph/0910.3670)

Johnson, J. A., Gaido., B. S., Sumi, T., Bond, I. A., \& Gould, A. 2008, ApJ, 685,508

Johnson, J. A., Gal-Yam, A., Leonard, D. C., Simon, J. D., Udalski, A., \& Gould, A. 2007, ApJ, 655, L3

Kane, S. R., \& Sahu, K. C. 2006, ApJ, 637, 752

Kim, Y.-C., Demarque, P., Yi, S. K., \& Alexander, D. R. 2002, ApJS, 143, 499

Lennon, D. J., Mao, S., Fuhrmann, K., \& Gehren, T. 1996, ApJ, 471, L23

Lennon, D. J., Mao, S., Reetz, J., Gehren, T., Yan, L., \& Rezini, A. 1997, ESO Messenger, 90, 30

Luck, R. E., Kovtyukh, V. V., \& Andrievsky, S. M. 2006, AJ, 132, 902

Poindexter, S., Afonso, C., Bennett, D. P., Glicenstein, J.-F., Gould, A., \& Szymanski, M. K. 2005, ApJ, 633, 914

Rich, R. M., Origlia, L., \& Valenti, E. 2007, ApJ, 665, L19

Udalski, A. 2003, Acta Astron., 53, 291

Udalski, A., et al. 2005, ApJ, 628, L109

Vogt, S. E., et al. 1994, Proc. SPIE, 2198, 362

Zocalli, M., Hill, V., Lecureur, A., Barbuy, B., Renzini, A., Minitti, D., Gomez, A., \& Ortolani, S. 2008, A\&A, 486, 177 